\title{
Prostaglandins: methodology
}

\author{
Brian J. Millard \\ B.Sc., Ph.D. \\ Institute of Neurology, London
}

\section{Introduction}

The recent history of prostaglandin measurement has been one of continual improvement, both in sensitivity and specificity. In the late 1960s, bioassay was the most widely used method of assay (Vane, 1969), and although a not very specific technique, was capable of measuring sub-nanogram quantities. Since then, a number of assays have been developed, such as enzymatic, specific binding, spectrofluorimetric, radioimmunoassay and gas chromatography, the latter especially in combination with mass spectrometry. The most widely used at present are radioimmunoassay (RIA) and gas-chromatography mass spectrometry (GCMS), and the advantages and disadvantages of these methods are discussed.

\section{Discussion}

The method of radioimmunoassay was first described in 1960 (Yalow and Berson, 1960) for the determination of insulin. In the prostaglandin field, an RIA for prostaglandin $F_{2 \alpha}$ appeared 10 years later (Levine and Van Vanukis, 1970). Since then, RIAs have been developed for the commonly encountered prostaglandins $F_{1 \alpha}, E_{1}, E_{2}, A_{2}$ and $B_{2}$. The method depends upon the competition of radioactively-labelled antigen (with tritium or ${ }^{125} \mathrm{I}$ ) with unlabelled antigen for a specific antibody site. The amounts of antibody and radioactive antigen are standardized so that the degree of binding of the labelled antigen is only affected by the quantity of unlabelled antigen added to the system.

RIAs have the advantage of being cheap and a sensitivity of the order of tens of picograms. They can be used upon the biological sample without the need for extraction or the formation of derivatives. The method is therefore extremely attractive, and is widely used at present. The major disadvantage is the lack of specificity. Thus anti-PGF $2 \alpha$ serum can react with both $\mathbf{P G F}_{2 \alpha}$ and $\mathrm{PGF}_{1 \alpha}$, while anti-PGB ${ }_{2}$ serum can react with $\mathrm{PGE}_{1}, \mathrm{PGE}_{2}$, $\mathrm{PGB}_{1}, \mathrm{PGB}_{2}$ and $\mathrm{PGA}_{2}$. Because of this, the results of an RIA are best presented as ' $\mathrm{PGF}_{2 \alpha}$ equivalents' and so on. The results of an RIA are best checked periodically by the virtually $100 \%$ specific GCMS method, so that the specificity can be determined.

The use of GCMS for the estimation of low levels of prostaglandins was pioneered by Samuelsson, Hamberg and Sweeley (1970). They introduced the concept of a stable isotope labelled analogue of the particular prostaglandin to act as a carrier through the extraction and derivatization procedure, and through the gas chromatographic column. By this means, it was argued that small amounts of the unlabelled prostaglandin which would otherwise be lost by adsorption on the column would be carried through, thus increasing the sensitivity of the method to the sub-nanogram range. In addition, the carrier, since it has a different molecular weight and fragmentation pattern, can be used as an internal standard.

However, it has been shown recently that it is possible to measure low levels of $\mathrm{PGF}_{2 \alpha}$ down to 50 femtograms per injection on the column without a carrier if the column is properly conditioned (Cory et al., 1976; Wilson et al., 1975). Work in this laboratory has now shown that tetradeuterated prostaglandins, contrary to previous reports, do not act as carriers through the GCMS system for small amounts of the unlabelled material, and therefore it is preferable to use cheaper homologues of the prostaglandin as internal standards for the analysis. These are chosen so as to give an intense fragment ion in common with the prostaglandin under study, so that the mass spectrometer can be used in its most sensitive mode of single ion monitoring.

\section{References}

Cory, H.T., Lascelles, P.T., Millard, B.J., Snedden, W. \& Wilson, B.W. (1976) Determination of prostaglandin $\mathrm{F}_{2 \alpha}$ by single ion monitoring. Biomedical Mass Spectrometry, 3, 117.

LeVINe, L. \& VAN VANukis, H. (1970) Antigenic activity of prostaglandins. Biochemical and Biophysical Research Communications, 41, 1171. 
Samuelsson, B., Hamberg, M. \& Sweeley, C.C. (1970) Quantitative gas chromatography of prostaglandin E1 at the nanogram level: use of deuterated carrier and multiple-ion analyser. Analytical Biochemistry, 38, 301.

VANE, J.R. (1969) The release and fate of vaso-active hormones in the circulation. British Journal of Pharmacology, $12,344$.
Wilson, B.W., Snedden, W., Tham, K.T. \& Willoughby, D.A. (1975) Future Trends in Inflammation, p. 25. Birkhauser Verlag, Basel.

YALOW, R.S. \& BerSON, S.A. (1960) Immunoassay of endogenous plasma insulin in man. Journal of Clinical Investigations, 39, 1157. 